\title{
Diagnosis of Phobic Anxiety Disorders using Virtual Reality Environment
}

This paper was downloaded from TechRxiv (https://www.techrxiv.org).

\section{LICENSE}

CC BY 4.0

SUBMISSION DATE / POSTED DATE

$10-02-2022$ / 11-02-2022

\section{CITATION}

Munir, Alina; Saleem, Dr. Yasir (2022): Diagnosis of Phobic Anxiety Disorders using Virtual Reality Environment. TechRxiv. Preprint. https://doi.org/10.36227/techrxiv.19153769.v1

$\mathrm{DOI}$

10.36227/techrxiv.19153769.v1 


\title{
Diagnosis of Phobic Anxiety Disorders using Virtual Reality Environment
}

\author{
Alina Munir, Member, IEEE, and Yasir Saleem, Supervisor, IEEE
}

\begin{abstract}
Specific Phobia Anxiety disorders are rarely detected in a clinical setting and are indicators of other grave mental health problems. VR is a persuasive tool for diagnosing and treating psychiatric problems, but the Virtual Reality applications developed are reported to lack a methodological approach. This particular research consults the Diagnostic and Statistical Manual of Mental Health Disorders (DSM-5) by the American Psychiatric Association (APA)to strengthen objectivity and add reliability to the assessment. We investigated participants' behavior in the Virtual Reality Environment. The participant walks or runs along a road in four different scenarios of a Virtual Reality Environment. The first scenario is a neutral environment with no phobic stimulus; the afterward situations pose for a dog cue, thunder lightning stimulus, and a combination of both stimulation consecutively. The assessment measures include distance and time calculated in each VRE scene, minimum and maximum oxygen levels and heart rate recorded for each scenario, physical and verbal reactions observed, self-reported measure of Cybersickness, and Sense of Presence. Participant's information such as name, age, gender, any bad experience with dogs or thunder lightning, and proposed phobia severity levels of parents. The "APA Specific Phobia Severity Measure - Adult" by DSM-5 classifies every record. Applying Artificial Intelligence learning models suggests Astraphobic patients also suffer from Dog phobia. The results imply that the Sense of Presence is a decisive factor in the assessment. It signifies that distance, time interval, and change in oxygen levels in the presence of phobic cues are influential parameters for phobia estimation.
\end{abstract}

Index Terms-Virtual Reality, Assessment, Cynophobia - Dog Phobia, Astraphobia - Thunder-lightning phobia, Data Mining.

\section{INTRODUCTION}

A NXIETY DISORDERS is one of the most common mental health problems worldwide. According to 2017 statistics, an estimated 284 million people suffer from anxiety disorders [1]. Specific Phobia Anxiety disorder is one of the eleven types of Anxiety disorder, Figure 1, in which phobic stimulus almost always arouses immediate fear or anxiety that is out of proportion to the actual threat considered in that culture. Phobia stimulus has further been classified into following classes: Animal (300.29 (F40.218)); Natural environment (300.29 (F40.228)); Blood-injectioninjury (300.29 (F40.23X)); Situational (300.29 (F40.248)); and "Other" (300.29 (F40.298)). This anxiety disorder adversely affects interpersonal relations, educational performance, or professional growth. These patients are $60 \%$ more likely to attempt suicide. In the absence of other mental problems, these phobias are detected rarely in a clinical setting. They prevail in combination with other mental disorders such as Personality Disorders and other types of Anxiety Disorders. Phobia develops in a person who went through or witnessed a traumatic incident, had an unexpected panic attack, or watched extensive media coverage of a harrowing event. It may develop genetically or due to behavioral inhibition and surrounding environmental conditions. It usually develops in early childhood. The patients mostly don't remember the reason for their phobia development. Approximately 75\% of specific phobic patients are anxious about more than one

- A. Munir is with the Department of Computer Engineering, University of Engineering and Technology, Lahore, 54890. E-mail:alinmunir@gmail.com

- Y. Saleem is with the Department of Computer Engineering, University of Engineering and Technology, Lahore, 54890.

E-mail: yasir@uet.edu.pk

Manuscript received Month day, 20XX; revised Month day, 20XX.

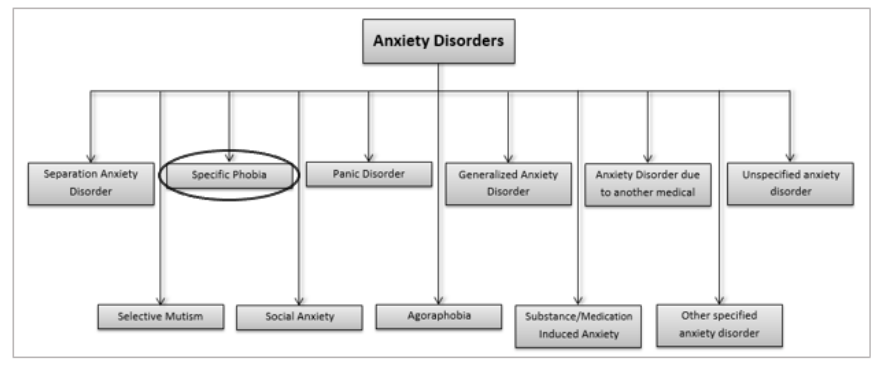

Fig. 1. Types of Anxiety Disorders

object or situation. There is a high possibility for the patient to develop Depressive Disorders, Bipolar and Related Disorders, Somatic symptoms and related Disorders, Substancerelated Disorders, and other Anxiety Disorders [2].

Predominantly, the diagnosis of psychiatric disorders is supported by clinical interviews and questionnaires. Psychiatrists have difficulty diagnosing when their sessions are based entirely on questions-answers and self-reported measures that a person has to recall. Also, there is no proof of how the patient will behave in a real scenario when there is a high certainty that shared details may be biased intentionally or unintentionally [3], [4], [5]. Real-world scenarios implementation for assessment analysis has security risks and immense financial constraints. Some environments are impossible to create in real for phobia severity diagnosis. Today, Virtual Reality technology renders as a potential tool in psychiatry. It has the potential to strengthen objectivity and add reliability in the diagnosis of psychiatric disorders. It may alleviate overcoming availability and accessibility problems of psychological help to people. 
The role of VR differentiates between diagnosis and treatment of Psychiatric Disorders. The applications developed for mental health disorders are reported not up to the mark. There is a big room for improvement in methodological studies conducted [6]. This research is designed according to standards described in the Diagnostic and Statistical Manual of Mental Health Disorders- Fifth Edition (DSM5). DSM-5 is a taxonomic and diagnostic tool by American Psychiatric Association (APA). It is considered the gold standard and has immense practical significance globally. The objective of diagnosing Cynophobia and Astraphobia is achieved by prototyping predictable, reliable, and safe test environments using modern and dependable technology: Virtual Reality. "VR is real-time and controlled computergenerated 3D graphics with user trackable multi-sensory interface that gives the user the experience of interaction and immersion in Virtual Environment" [6], [7]. The VR industry is gaining momentum; its market size was 4.8 billion US dollars in 2021 and is projected to expand more [8].

This paper describes the Virtual Reality Environment design to segregate phobic and non-phobic participants to Dog and Thunder-lightning stimuli. The VRE manipulates participant behavior in four different scenarios; neutral scenario, environment with dog stimulus, thunder lightning stimulus, and both pre-mentioned stimuli. The participant wears the headset and moves virtually in the VRE with the help of a remote Bluetooth controller. The developed VR application for the mobile android device calculates and saves the distance and time between two fixed checkpoints for each scenario in a file. Pulse-oximeter measures heart rate and oxygen for each setting. The researcher observes the exhibited physical and verbal reactions during VR exposure. Later, the participant fills the survey form with readings, observations, and relevant personal background. The "APA Specific Phobia Severity Measure - Adult" questionnaire classifies each record. The recorded experiments instances via Google form converted into the suitable format for Data Mining with the WEKA tool. The subsets of distinguishing features for applying learning models select through various attribute evaluators on the pre-processed data. The results are analyzed statistically: in terms of correctness (percentage correctness), accuracy (F-measure), agreeability (kappa statistic), and diagnostic separability (ROC Area). The results yielded support that Cynophobia and Astraphobia are highly correlated. The study also verifies the relevant literature and proposes distinct features for predicting severity levels of Cynophobia and Astraphobia. This study attempt to help save precious lives, add reliability to the diagnosis of mental problems and help standardize psychiatric diagnostic procedures.

\section{Literature Review}

VR environments have been implemented in the assessment of social behavior [9], Paranoia, ADHD (Attention-Deficit Hyperactivity Disorder), Autism, substance use disorders, and eating disorders. It investigated the assessment of cognitive disabilities of Schizophrenia patients. It has been used in forensics by evaluating psychological responses to certain virtually created situations. It is adopted for the evaluation of anxiety disorders such as tunnel phobia, PTSD (PostTraumatic Stress Disorder), panic disorder, social anxiety, and OCD (Obsessive Compulsive Disorder). [5] VR has been employing in treating PTSD, anxiety and specific phobias [3] [10] [11] , chronic pain, rehabilitation, and addiction [12]. Successful treatment of Specific Phobias using VR includes Arachnophobia (Spider Phobia), Katsaridaphobia (Cockroach Phobia), Cynophobia (Dog Phobia) [13] and AreoPhobia (Flying Phobia) [14]. VRET (Virtual Reality Exposure Therapy) is applied to treat different types of Anxiety Disorders such as Agoraphobia, Panic Disorder, and Social Anxiety Disorder. It is adopted to treat Schizophrenia and Autism [15].

I Viaud-Delmon et al. [16] validated audio-visual receptory information in VR add immersion and realism. Clara Suied et al. [13] also studied the effects of auditory-visual perceptions on the sense of presence and emotions for virtually created cynophobic scenarios. The study suggests a direct correlation between the sense of presence in VE and arousal of emotional response. Research advised that Physical attributes such as size and color, and animated behaviors such as standing, laying down, growling, and barking of dog are decisive in stimulating fear reactions. Fearful participants responded verbally and physically to the phobic stimulus. The study reveals audio adds realism and immersion to the scene but only when these two perceptions i.e. audio and visual, are synchronized. Marine Taffou et al. [17] studied the effect of multisensory information in provoking fearful emotions. During experimentation, selfreported distress was recorded in VE by measures and skin conductance through a wireless SCL device. Members also proclaimed Cybersickness. Observed readings suggested that aversive stimulus may influence distance perceptions. The study also proposed that controlling factors over the situation also impact fear reactions. An aversive stimulus is more likely to affect fearful persons than non-fearing individuals. This investigation submitted that multisensory presentation embellishes the conscious experience of emotions.

Marine Taffou et al. [18] studied that patients suffering from Specific Phobia Anxiety Disorders, especially, Cynophobia show less reaction time to phobic stimulus as compared to non-fearing individuals. They anticipate less distance between them and phobic stimulus compared to actual distance, thus causing annoyance and defensive behaviors such as flight. This underestimation of distance from phobic stimulus directly affiliates with a person's anxiety level. Anxiety fails a person to synchronize space perception and concrete form. Marine Taffou et al. hypothesized that phobic stimulus expands peripersonal space i.e. adjacent area encircling our bodies to protect ourselves.

Narit Hnoohom et al. [19] designed a virtual reality smartphone application for users to encounter their dog phobia. The experimental setup consisted of an android mobile embedded with a gyroscope sensor, full-size headphones, and HMD. A computer-generated 3D graphical environment was developed for VR using the Unity3D platform. The reactions of a virtual dog were designed to be triggered accordingly to the units of distance settled between user and dog. VR surroundings are secured and easily accessible to learn to defy phobias. Jerdan SW. et al. 
[20] highlights that VR with mobile head-mounted devices is getting popular with being affordable and available. It highlights the service of VR concerning mental health problems and its adoption for Anxiety, Pain and Stress Management, Depression, Eating Disorders, Addiction, and Substance Abuse therapies.

Alexander Yu. Tychkov et al. [21] reviewed and critically analyzed methods and tools for specific phobia anxiety disorders assessment. It suggested heart-rate variation is a physiological reaction to stress stimuli. It emphasizes EEG patterns of frequency rhythms alpha/beta ratio decreases whereas theta rhythm amplifies under phobic disorders. EEG is brain neurons' response to an external stimulus. Alpha, beta, theta, and delta are EEG signals. It also points out the importance of verbal and non-verbal reactions as an indicator and the standard questionnaire for evaluation. It stresses the methodological gap for the developed VR environments to patient's needs. It insists on parameters measuring brain and heart signals for evaluating phobic disorders. Kody Wood et al. [22] investigates that physiological measures such as pulse rate, galvanic skin responses, gaze, brain, verbal, and non-verbal activity play a vital role in better assessment and improvement of user experience. The massively adopted modern and advanced wearable devices have enabled the integration with VR technology for measuring physiological behaviors. It critically analyzes literature relevant to Assessment and Monitoring, Customization, and Adaptability using physiological measures in VR. It mentions the importance of the Sense of Presence in the virtual environment and suggests immersive environments elicit strong emotional and physiological responses. The participants reported being fast in VR environments than in the real-world environment. Their brain and muscular physiological measures were determined using suitable devices. It also cites the solution suggested to improve motion sickness and cybersickness.

\section{Methodology}

The methodologies proposed for the experiment includes Virtual Reality Environment development for the diagnosis of Cynophobia and Astraphobia, assessment measures to consider, and use of Data Mining to extract useful information and patterns.

\subsection{Virtual Reality Environment Development}

Hardware devices employed for experimenting VRE include VR headset glasses, VR Bluetooth remote controller and android device. VRE environment is developed using a computer system with appropriate and recommended installed softwares Java Development Kit, Android Studio, Unity 3D, Visual Studio and Blender.

VR Headset viewer used in experiment is BOBOVR $Z_{4}$. It features adjustable knobs for pupil and diopter adjustments. It has Japan imported optical PMMA lens with 35mm focal length and $120^{\circ}$ FOV. It consists of integrated visual and auditory systems. It facilitates phones with size range from $4^{\prime \prime}$ to $6^{\prime \prime}$. It is designed ergonomically with a head belt for supporting VR glasses and a semi-transparent heatdissipating cover design. VR Bluetooth Remote Controller acquires support for iOS, Android, and PC. It features $360^{\circ}$ joystick rotation, mode selection key, function keys, power, and trigger buttons [23]. Android Device running an OS with the minimum of version 4.4 (API level 19) is required [24]. This experiment is performed using devices running Android API levels 27 and 28. It is requisite for an Android Device equipped with a Gyroscope sensor, as this sensor helps create a presence of self in VRE [25]. Frame rate below 90 frames per second creates cybersickness. Therefore the application is programmed to operate with 90 frames per second. JDK is a development environment utilized by Android Studio [26]. Android Studio is the official IDE for building apps for every type of Android device [27]. Unity $3 \mathrm{D}$ is a real-time development platform for creating games and immersive environments, Version: 2018 adopted for this experiment [28] - Figure 2. Visual Studio is IDE for developing and editing code for different platforms. Here, it is used for writing scripts for the Unity platform [29]. Blender is a free and open-source 3D software, its 2.9 version opts for rigging and animation of character model [30] - Figure 3.

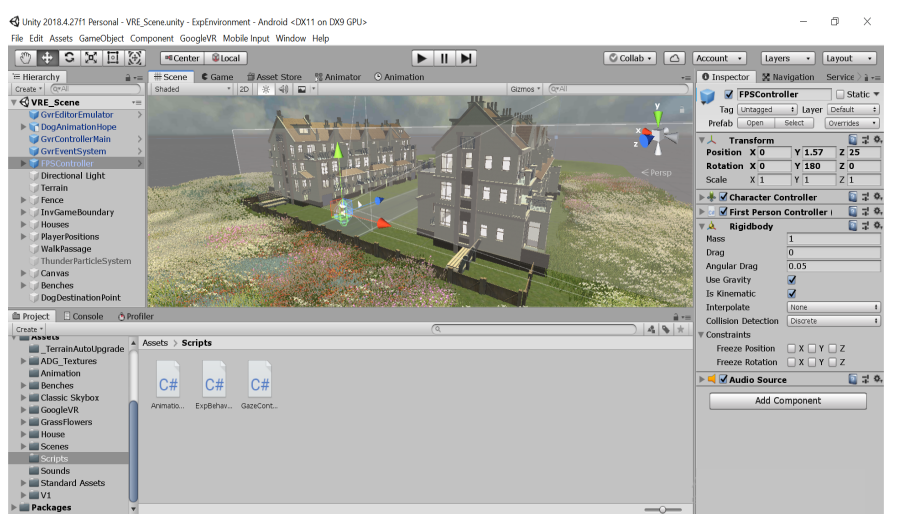

Fig. 2. VRE development in Unity 3D

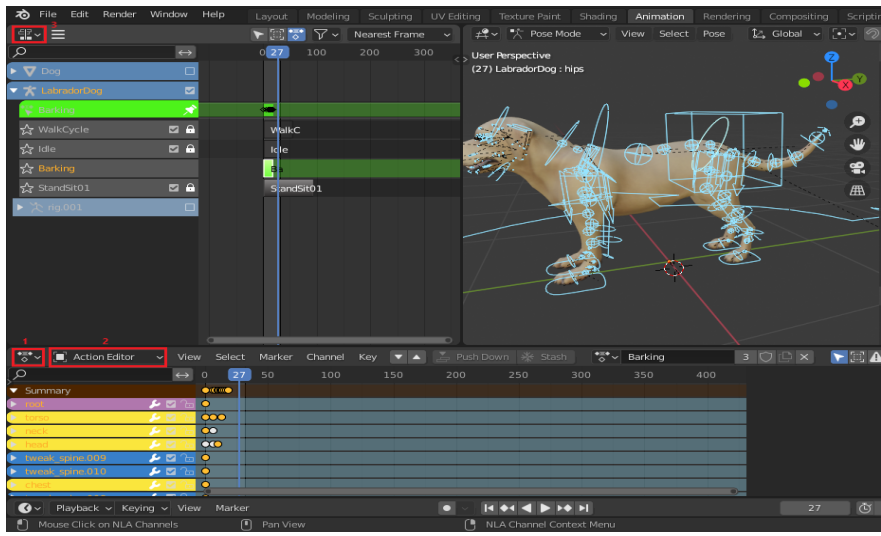

Fig. 3. Dog Animation in Blender 2.9

\subsubsection{Idea}

The idea behind the game is that participant has to move from a starting point to ending point areas in an outdoor VR environment under four different circumstances. VRE measures the distance and time to reach the endpoint in the vicinity of the impending feared object. Scenarios selected for environment development are listed as follows: 
- A neutral scenario without dog and thunder lightning - Figure 4.

- A scheme with a dog walking and barking animations - Figure 5.

- A thunder lightning set-up - Figure 6.

- Integrating the last two mentioned scenarios for studying the combined effect - Figure 7.

\subsubsection{Guidelines}

Principles for this VRE are reflected as follows:

- There is no win or loss score for the setup. The application calculates the duration and the distance of finished and unfinished checkpoints under four different scenarios.

- Participants will always spawn in the same spot, the target mark will always be the same, and the default mode selected is walking.

- Player can run and walk in the scenarios, and the introduction to this option mimics the ability to run away in fearful situations.

- Players will be able to exit at any time in the Virtual Environment. This option adapts for safety, such as participants being unable to endure phobic stimulus or due to intolerable cybersickness.

- Each scenario will start with the participant's permission by gazing at the play button for a short interval of time (Figure 8 ) or by pressing a button, especially programmed, on the Bluetooth remote controller.

- There will be a sequence of scenarios represented to the player. The experiment starts from the neutral scenario to the cynophobic situation, it then advances to the astraphobic setup, and adheres to the combination of both cynophobic and astraphobic environments.

- Objective of this game is to get time and distance covered for completed checkpoints for each participant's reactions to phobic and non-phobic cues.

\subsection{Assessment Measures}

The assessment measures for the diagnosis of Cynophobia and Astraphobia include the following: distance and time calculated in each VRE scene, minimum and maximum oxygen levels and heart rate recorded for each scenario, physical and verbal reactions observed, self-reported measure of $\mathrm{Cy}$ bersickness and Sense of Presence. Participant's information such as name, age, gender, any bad experience with dogs or thunder lightning, and proposed phobia severity levels of parents. The "APA Specific Phobia Severity Measure Adult" by DSM-5 to classify every record for diagnosis parameters. All the measures are collected and managed through Google form and explained in the following paragraphs [31]. Figure 9 pictures the survey summary.

Attributes Name, Age and Gender are selfexplanatory. MotherPhobia_Dog, FatherPhobia_Dog, MotherPhobia_Thunder, and FatherPhobia_Thunder are the suggested parents' phobia severity levels by the participant. These levels are None, Mild, Moderate, Severe, and Extreme in ascending order. BadExperience_Dog and BadExperience_Thunder depict query as any bad experience with the dog or thunder lightning.
HometownArea symbolize Rural or Urban home town. DSMAstraphobia and DSMCynophobia express APA severity measure for Adult Phobia [32]. American Psychiatric Association has established procedures and potential tools for research and clinical evaluation of adult-specific phobia severity measures.

Reactions outline the overall feelings during the experiment. It's an option of Calm, Uneasy, or Little uneasiness. Re_HeartRateVariation, Re_Shaking, Re_ShortnessOfBreath, Re_Nausea, Re_Sweating, and Re_Crying characterize the reactions observed or felt as variations in Heart Rate, shaking or trembling, shortness of breath or difficulty in breathing, sensation of vomiting, sweating, and crying, respectively. TotalReactions indicates the total sum of the previously mentioned six reactions. phobicStimulus identify intimidating stimulus in the VRE. It is either dog, thunder-lightning, or both. CyberSickness indicates the state level of dizziness aroused by wearing the VR headset. It is None, Mild or Strong. VRPresence portrays the Good, Moderate, and Bad feeling of presence in the VR environment. LevelQuit outlines the level quitted due to cybersickness or phobic stimulus. Zero (0) means the participant did not withdraw.

The features DistanceNeutral, TimeNeutral, VelocityNeutral, MinHRNeutral, MaxHRNeutral, DistHR_BothNeut, MinO2Neutral, MaxO2Neutral, and DistO2_BothNeut construes the distance travelled virtually from spawning to finish line, time taken, velocity, minimum Heart Rate, maximum Heart Rate, euclidean distance of former mentioned attribute Heart Rate (min, max) from Heart Rate values of Both stimuli(min, max), minimum oxygen level, maximum oxygen level, and euclidean distance of Oxygen level (min, max) from Oxygen level values of Both stimuli(min, max) for the neutral scenario. DistanceCyno, TimeCyno, VelocityCyno, MinHRCyno, MaxHRCyno, DistHR_BothCyno, MinO2Cyno, MaxO2Cyno, and DistO2_BothCyno support the prior interpretation for the Cynophobia assessment setup. Similarly, DistanceAstro, TimeAstro, VelocityAstro, MinHRAstra, MaxHRAstra, DistHR_BothAstra, MinO2Astra, MaxO2Astra, and DistO2_BothAstra advocates for Astraphobic VRE. DistanceBoth, TimeBoth, VelocityBoth, MinHRBoth, MaxHRBoth, MinO2Both, and MaxO2Both image for distance, time, velocity, minimum Heart Rate, maximum Heart Rate, minimum oxygen and maximum oxygen for the VRE with both dog and thunderlightning stimuli. This scenario serves as the oxygen and heart rate baseline measurement for the single stimulus setups.

Data Response statistics elaborate that $57 \%$ of participants felt a good VR presence, $66 \%$ were calm, $70 \%$ did not feel cybersickness. The $62 \%$ of participants were Females. The majority from urban hometown areas took part in the experiment. A total of 37 participants reported having a bad experience with the dog, whereas 18 participants reported a rough go through with thunder lightning. The majority suggested the mother phobia severity levels be higher than father phobia severity. The raw Cynophobia Dataset consists of 36 instances classified with "None", 43 with "Mild", 16 with "Moderate", 4 with "Severe" and 1 with "Extreme" phobia severity level. The raw Astraphobia Dataset subsists 


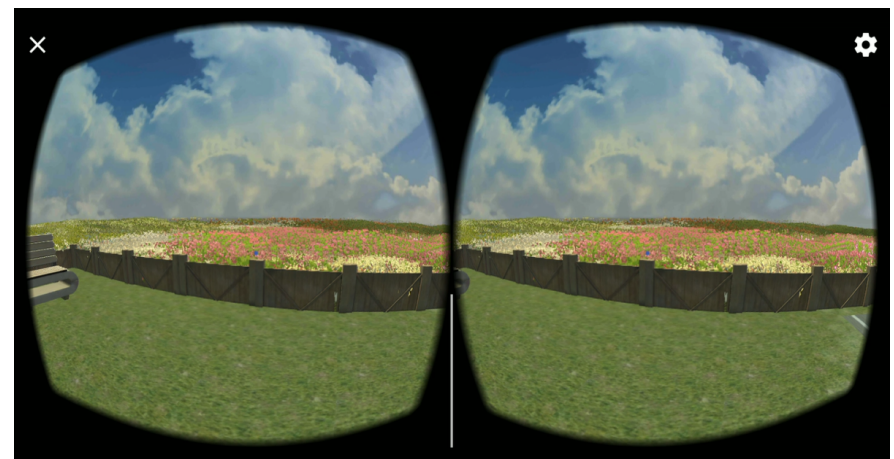

(a)

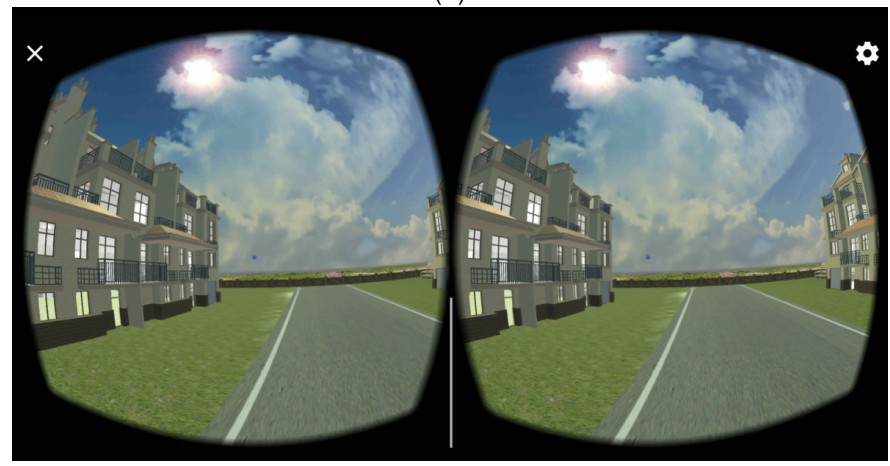

(b)

Fig. 4. VRE - Neutral Scenario

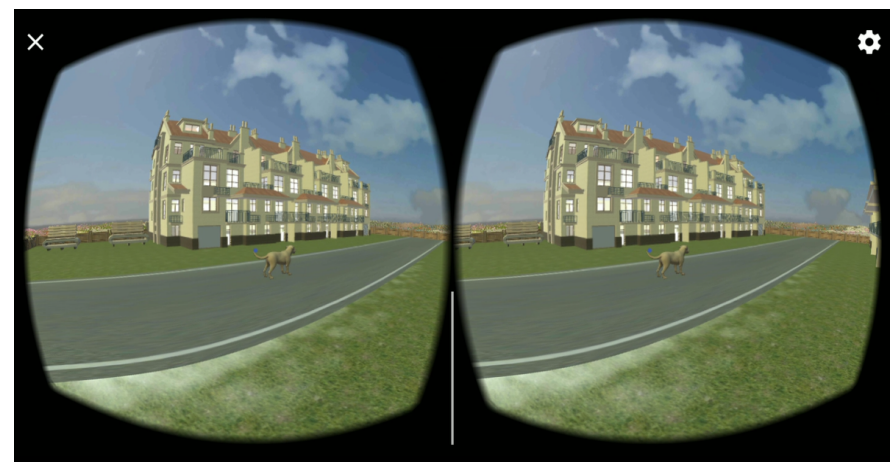

(a)

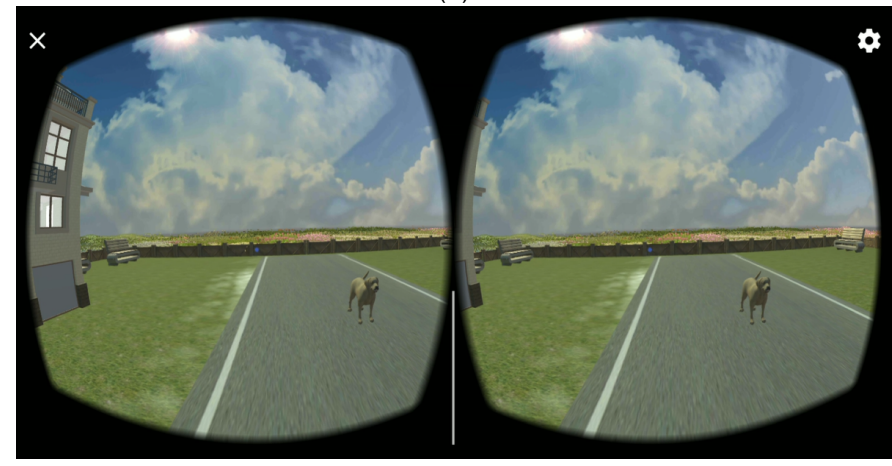

(b)

Fig. 5. VRE - Dog Scenario

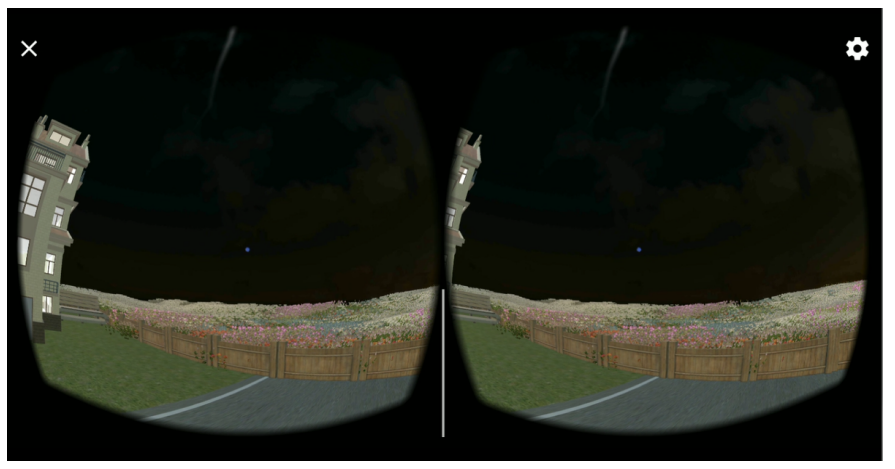

(a)

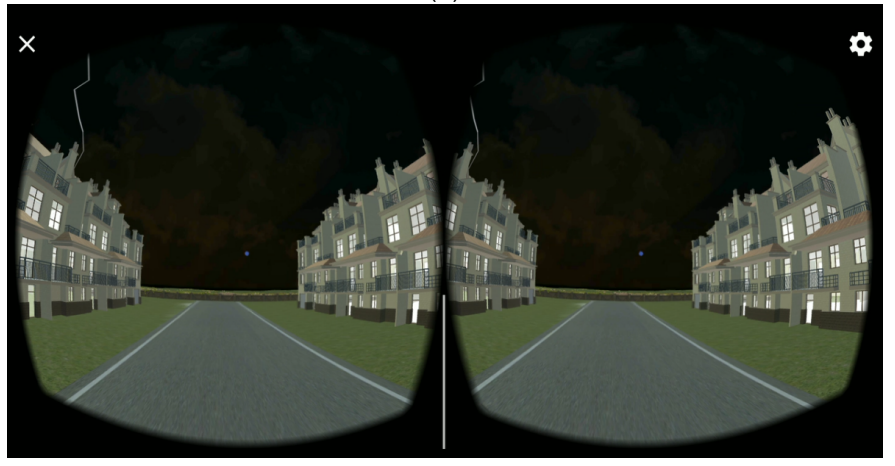

(b)

Fig. 6. VRE - Thunder Lightning Scenario

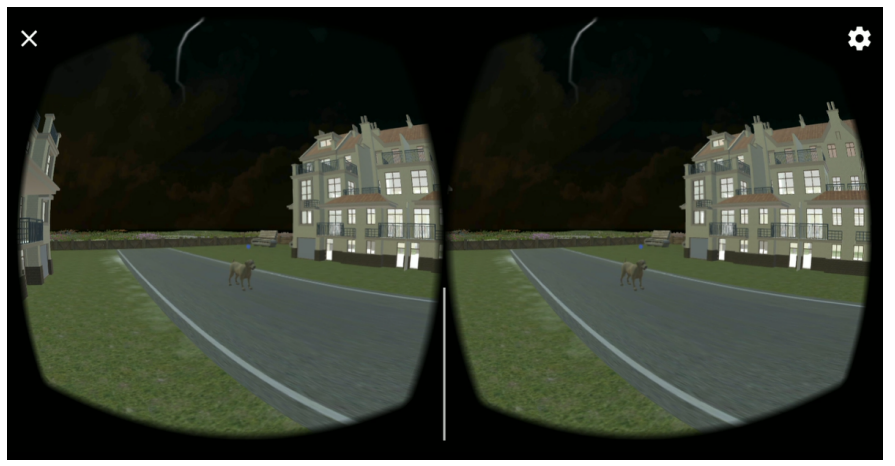

(a)

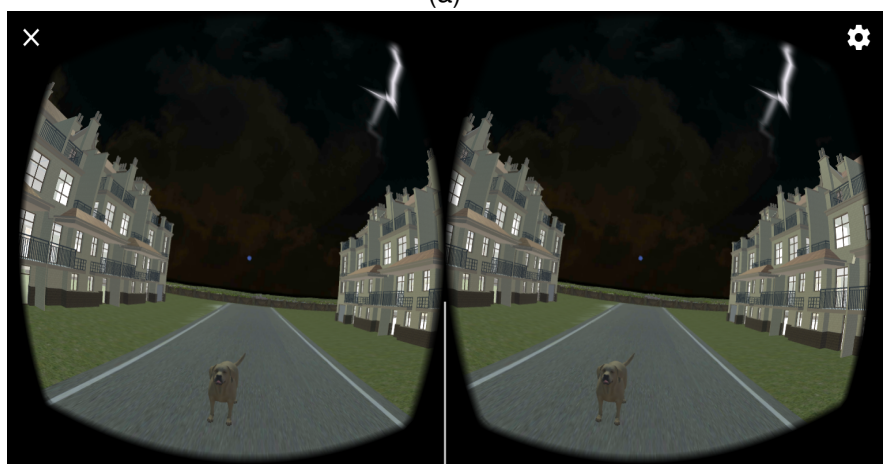

(b)

Fig. 7. VRE - Dog and Thunder Lightning Scenario 


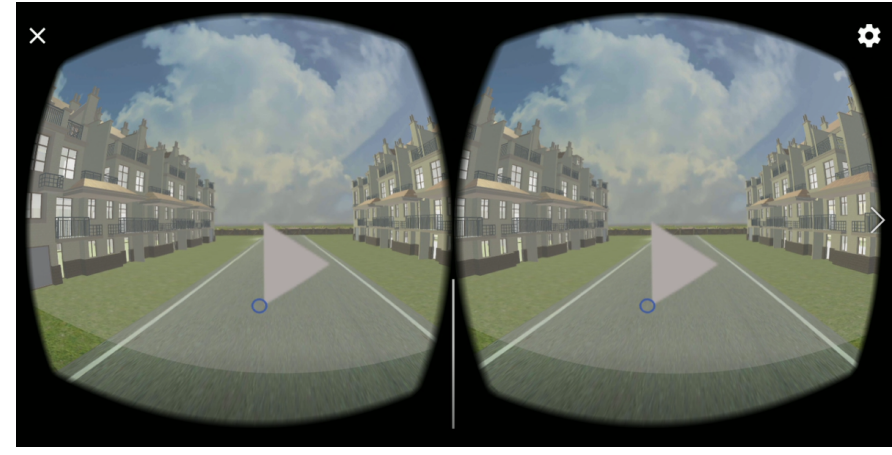

Fig. 8. Gaze Timer for Participant's permission in VR scenario

68 instances classified with "None", 25 with "Mild", 3 with "Moderate", 4 with "Extreme" and 0 with "Severe" phobia severity level. The classification is based on DSM-5 APA phobia severity level - Adult.

\subsection{Data Mining}

The final step is the procedure of Data Mining to the collected survey data. Data Mining is the process of getting valid, understandable, and beneficial information from the raw data. It entails a thorough understanding of the problem, real-world Data Collection, Feature Engineering, Data Cleaning, Model Selection, Evaluation of the models, and extracting valuable information from results. It is the application of Machine Learning. The participants above 18 years of age participated in the study. The raw dataset is converted to ARFF format, and the process of knowledge discovery is implied through the tool "Waikato Environment for Knowledge Analysis" acronym of "WEKA" [33].

The data consists of 100 instances and 47 distinct attributes before data preprocessing. A popular expression in data science, "Garbage In, Garbage Out" frames the significance of data preprocessing. The Web Document is filled under supervision to ensure correctness to the best of one's ability. The String attribute"Name" is removed as it has no impact on the results. The newly derived features that may improve the results are introduced, making 57 distinct attributes in the dataset. DSMCynophobia (Figure 9k) and DSMAstraphobia (Figure 9l) attributes are taken as a Class parameter and learning parameter alternatively for diagnosis and inter-relevancy of Cynophobia and Astraphobia. Both data sets do not have any redundant data or duplicated data. The unique or diminished class valued records are eliminated, addressing the overfitting problem. The data is randomized, statistically standardized, and discretized using WEKA filters.

Dimensionality Reduction improves the performance of the learning model. It involves the elimination of insignificant, irrelevant, or redundant attributes. The different methods opted for attributes selection are scheme-independent, ranking-based, and wrapper classifier for attribute selection approach. A set of Rules, Decision Trees, Bayesian Network, Support Vector, and Ensemble learning classifier models opt with the attributes selected by prior mentioned methods. 10-fold stratified Cross-Validation is a standard evaluation technique, opted for this research, as well. The performance of the learning model is statistically analyzed.
Gender

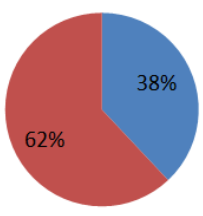

(a) Gender

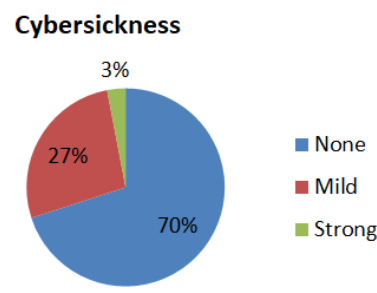

(c) Cybersickness

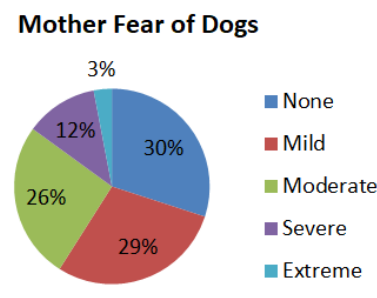

(e) Mother Phobia - Dog

Mother Fear of Thunder Lightning

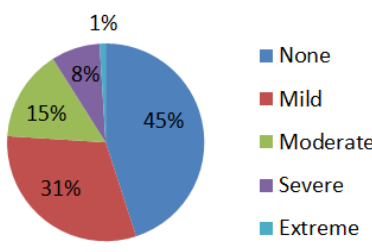

Bad Experience with Dog
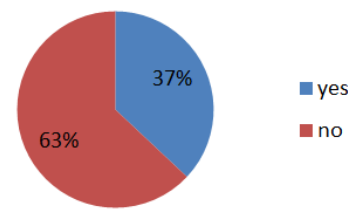

no

(i) Bad Experience - Dog

DSMCynophobia

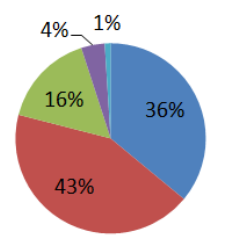

None

Mild

Moderate

- Severe

Extreme

(k) Cynophobia Severity Levels (g) Mother Phobia - Thunder
Overall Reaction

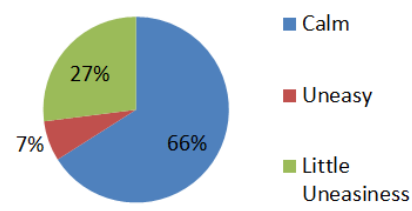

(b) Overall Reaction

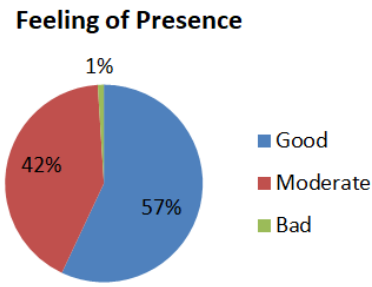

(d) VR Presence

Father Fear of Dogs

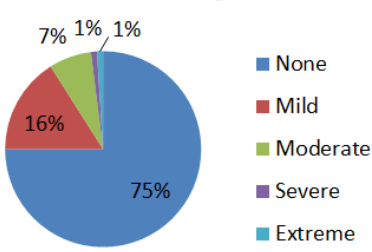

(f) Father Phobia - Dog

Father Fear of Thunder Lightning $4 \% \_1 \% 0 \%$

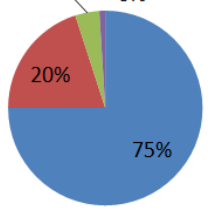

None

aild

Moderate

- Severe

Extreme

(h) Father Phobia - Thunder

Bad Experience with Thunder Lightning

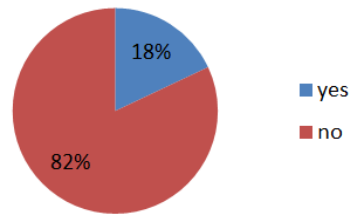

(j) Bad Experience - Thunder

DSMAstraphobia

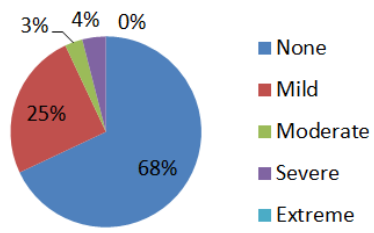

(I) Astraphobia Severity Levels
Fig. 9. Survey Statistics 


\section{Results}

The set of methods used for feature selection of Cynophobia and Astaphobia Datasets are as follows: Scheme independent method CfsSubsetEval; ranking based approach CorrelationAttributeEval, InfoGainAttributeEval and wrapper classifier method WrapperSubsetEval. The Wrapper learning techniques are NaiveBayes, IBk, K-Star, RandomTree, and J48. The classifiers: ZeroR, OneR, JRip, J48, REPTree, Naïve Bayes, IBk, SMO, and Bagging are applied to each set of selected attributes by each evaluator. ZeroR (baseline), OneR, and JRip are rule-based learners. J48 and REPTree are decision-tree learning models. Naïve Bayes is the Bayesian network classifier, IBk is the instance-based model, SMO is the Support Vector Machine algorithm, and Bagging is the ensemble learning classifier. The metrics for comparing performance are Correctly Classified instances, Kappa Statistic, ROC Area, and F-Measure. The dataset with DSMCynphobia (Specific Phobia Severity level of the participant for DSM-5 questionnaire) as class attribute consists of 79 instances and 57 attributes taking DSMAstraphobia as a parameter. The dataset with DSMAstraphobia as a class attribute consists of 93 instances and 57 attributes with DSMCynophobia as contributing attribute. This strategy is applied to find the interrelation of both phobias.

CFS Subset Evaluator Method using Forward BestFirst Searching on the training set generates the subsequently mentioned results in Table 1 on both datasets. Correlation Filter and Info Gain feature supervised evaluator using Ranking Search Method ranked top ten (10) attributes for Cynophobia and Astraphobia assessment as mentioned in Table 2 and Table 3, correspondingly. The subsets of the first five (5) and first nine (9) features for Correlation and Info Gain are selected, respectively, with the hit and trial method for obtaining better performance. Wrapper Subset Method evaluates attribute sets by adopting a learning scheme. Forward Best First searching method for attribute selection using Naive Bayes, IBK, KStar, Random Tree, and J48 algorithms. Table 4 represents the comparison measurements for both phobias.

The Table 5 and Table 9 represents the comparison of Correctly Classified Instances Percentage, Table 6 and Table 10 characterize Kappa Statistic for the applied classifiers on selected attributes subsets, Table 7 and Table 11 depicts calculation for the metric ROC Area, also known as AUC or Area Under ROC Curve, and Table 8 and Table 12 outlines F-Measure i.e. the information retrieval or accuracy of the model for Cynophobia and Astraphobia assessment, simultaneously. The comparison is also pictured in Figure 10 and Figure 11.

\section{Discussion}

This section discusses and analyzes the results and performance of learning models and attribute selection for Cynophobia and Astraphobia severity levels diagnosis. Classifier ZeroR is used to compare the efficiency of any classifier.

The analysis of Cynophobia severity level prediction reveals that the most optimal combination in terms of correctness, accuracy, agreeability, and diagnostic separability is instance-based learning model IBk with scheme
TABLE 1

Scheme Independent Attribute Evaluator: CfsSubsetEval

\begin{tabular}{|c|c|c|c|}
\hline Dataset & $\begin{array}{c}\text { Total } \\
\text { Subsets } \\
\text { Evaluated }\end{array}$ & $\begin{array}{c}\text { Best } \\
\text { Subset } \\
\text { Merit }\end{array}$ & Selected Attributes \\
\hline Cynophobia & 518 & 0.185 & $\begin{array}{l}\text { Age } \\
\text { TimeNeutral } \\
\text { DistanceCyno } \\
\text { DistO2_BothCyno } \\
\text { DSMAstraphobia }\end{array}$ \\
\hline Astraphobia & 697 & 0.157 & $\begin{array}{l}\text { FatherPhobia_Thunder } \\
\text { BadExperience_Thunder } \\
\text { TimeNeutral } \\
\text { VelocityNeutral } \\
\text { DistanceCyno } \\
\text { TimeCyno } \\
\text { TimeBoth } \\
\text { VelocityBoth } \\
\text { DSMCynophobia }\end{array}$ \\
\hline
\end{tabular}

TABLE 2

Ranking Based Attribute Evaluator - Cynophobia Dataset

\begin{tabular}{|l|l|}
\hline \multicolumn{2}{|c|}{ Cynophobia Dataset } \\
\hline Correlation Selection & Info Gain Selection \\
\hline \hline 0.3248 DSMAstraphobia & 0.208643 TimeNeutral \\
0.2414 VRPresence & 0.125253 Age \\
0.2313 Gender & 0.121237 DistanceNeutral \\
0.2127 DistO2_BothNeut & 0.111695 VelocityNeutral \\
0.2113 Re_Shaking & 0.101907 DistanceCyno \\
0.203 DistanceNeutral & 0.097913 DSMAstraphobia \\
0.1976 FatherPhobia_Dog & 0.095145 VelocityBoth \\
0.1968 CyberSickness & 0.092905 TimeCyno \\
0.1865 TotalReactions & 0.092031 VelocityAstra \\
0.1829 TimeAstro & 0.070572 DistO2_BothCyno \\
\hline
\end{tabular}

TABLE 3

Ranking Based Attribute Evaluator - Astraphobia Dataset

\begin{tabular}{|l|l|}
\hline \multicolumn{2}{|c|}{ Astraphobia Dataset } \\
\hline Correlation Selection & Info Gain Selection \\
\hline \hline 0.2377 BadExperience_Thunder & 0.1794864 VelocityNeutral \\
0.22393 DSMCynophobia & 0.1310864 TimeNeutral \\
0.19977 FatherPhobia_Thunder & 0.1233133 VelocityBoth \\
0.19407 HometownArea & 0.0901844 TimeBoth \\
0.19315 phobicStimulus & 0.0849085 TimeCyno \\
0.17806 Re_ShortnessOfBreath & 0.0846677 DistanceNeutral \\
0.17195 Re_Crying & 0.0761319 VelocityAstra \\
0.17195 Re_Sweating & 0.075692 Age \\
0.17195 LevelQuit & 0.0735588 DSMCynophobia \\
0.15863 Gender & 0.0608173 DistanceCyno \\
\hline
\end{tabular}


TABLE 4

Wrapper Classifier Subset Attribute Evaluator - Cynophobia Dataset

\begin{tabular}{|c|c|c|c|c|c|c|}
\hline Dataset & Parameter & IBK & KStar & Naive Bayes & RandomTree & $\mathrm{J} 48$ \\
\hline \multirow[b]{3}{*}{ Cynophobia } & Total subsets Evaluated & 423 & 572 & 567 & 566 & 377 \\
\hline & Best Subset Merit & 0.797 & 0.785 & 0.787 & 0.787 & 0.747 \\
\hline & Selected Attributes & $\begin{array}{l}\text { VRPresence } \\
\text { MinHRCyno } \\
\text { DSMAstraphobia }\end{array}$ & $\begin{array}{l}\text { Re_Shaking } \\
\text { TotalReactions } \\
\text { VRPresence } \\
\text { MinHRBoth } \\
\text { DSMAstraphobia }\end{array}$ & $\begin{array}{l}\text { Age } \\
\text { VRPresence } \\
\text { DistO2_BothNeut } \\
\text { DistanceCyno } \\
\text { DistO2_BothCyno } \\
\text { DSMAstraphobia }\end{array}$ & $\begin{array}{l}\text { Re_ShortnessOfBreath } \\
\text { VRPresence } \\
\text { DistO2_BothCyno } \\
\text { MinHRBoth } \\
\text { MinO2Both } \\
\text { DSMAstraphobia }\end{array}$ & $\begin{array}{l}\text { VRPresence } \\
\text { DSMAstraphobia }\end{array}$ \\
\hline \multirow[b]{3}{*}{ Astraphobia } & Total subsets Evaluated & 528 & 974 & 659 & 424 & 276 \\
\hline & Best Subset Merit & 0.796 & 0.841 & 0.806 & 0.796 & 0.731 \\
\hline & Selected Attributes & $\begin{array}{l}\text { Re_HeartRateVariation } \\
\text { HometownArea } \\
\text { MaxHRCyno } \\
\text { MaxO2Astra }\end{array}$ & $\begin{array}{l}\text { Age } \\
\text { Gender } \\
\text { FatherPhobia_Thunder } \\
\text { Re_Nausea } \\
\text { TimeNeutral } \\
\text { DistHR_BothNeut } \\
\text { MinO2Neutral } \\
\text { MinO2Cyno } \\
\text { MaxHRAstra } \\
\text { MaxO2Astra } \\
\text { MinO2Both } \\
\text { LevelQuit }\end{array}$ & $\begin{array}{l}\text { Re_Sweating } \\
\text { TotalReactions } \\
\text { CyberSickness } \\
\text { HometownArea } \\
\text { MinHRNeutral } \\
\text { DistO2_BothNeut } \\
\text { VelocityAstra } \\
\text { TimeBoth }\end{array}$ & $\begin{array}{l}\text { DistanceAstro } \\
\text { VelocityBoth } \\
\text { DSMCynophobia }\end{array}$ & null \\
\hline
\end{tabular}

TABLE 5

Correctly Classified Instances Percentage - Cynophobia Dataset

\begin{tabular}{|c|c|c|c|c|c|c|c|c|c|c|}
\hline $\begin{array}{l}\text { Selected } \\
\text { Attributes }\end{array}$ & ZeroR & OneR & JRip & $\mathrm{J} 48$ & REPTree & Naive Bayes & IBk & SMO & Bagging & $\begin{array}{l}\text { Attributes } \\
\text { - Average }\end{array}$ \\
\hline All & 54.46 & 49.29 & 54.29 & 58.21 & 60.54 & 67.14 & 59.64 & 57.14 & 57.14 & 57.54 \\
\hline CFS & 54.46 & 56.79 & 59.29 & 56.96 & 58.21 & $\overline{59.64}$ & 64.64 & 64.46 & 65.54 & 60.00 \\
\hline Correlation & 54.46 & 44.11 & 57.86 & 64.64 & 56.43 & 49.46 & 55.71 & 52.68 & 51.25 & 54.07 \\
\hline InfoGain & 54.46 & 55.54 & 58.04 & 55.71 & 55.54 & 61.96 & 58.21 & 60.71 & 54.46 & 57.18 \\
\hline W-IBK & 54.46 & 45.54 & 53.04 & 60.71 & 58.21 & 57.14 & 58.57 & 62.14 & 60.54 & 56.70 \\
\hline W-KStar & 54.46 & 45.71 & 58.39 & 58.39 & 60.89 & 56.96 & 60.71 & 58.39 & 60.89 & 57.20 \\
\hline W-NaiveBayes & 54.46 & 45.71 & 58.39 & 60.89 & 49.46 & 61.07 & 52.14 & 60.89 & 53.04 & 55.12 \\
\hline W-RandomTree & 54.46 & 44.29 & 56.79 & 59.46 & 56.79 & 58.04 & 55.54 & 53.04 & 60.54 & 55.44 \\
\hline W-J48 & 54.46 & 45.71 & 62.14 & 62.14 & 62.14 & 64.64 & 62.14 & 62.14 & 62.14 & 59.74 \\
\hline Model-Average & 54.46 & 48.08 & 57.58 & 59.68 & 57.58 & 59.56 & 58.59 & 59.06 & 58.39 & \\
\hline
\end{tabular}

TABLE 6

Kappa Statistic - Cynophobia Dataset

\begin{tabular}{|c|c|c|c|c|c|c|c|c|c|c|}
\hline $\begin{array}{l}\text { Selected } \\
\text { Attributes }\end{array}$ & ZeroR & OneR & JRip & $\mathrm{J} 48$ & REPTree & Naive Bayes & IBk & SMO & Bagging & $\begin{array}{l}\text { Attributes } \\
\text { - Average }\end{array}$ \\
\hline All & 0.00 & 0.01 & 0.07 & 0.15 & 0.19 & 0.34 & 0.20 & 0.13 & 0.14 & 0.14 \\
\hline CFS & 0.00 & 0.16 & 0.16 & 0.15 & 0.15 & $\overline{0.19}$ & 0.30 & 0.28 & 0.33 & 0.19 \\
\hline InfoGain & 0.00 & 0.14 & 0.16 & 0.12 & 0.11 & 0.23 & 0.18 & 0.20 & 0.08 & 0.14 \\
\hline W-IBK & 0.00 & -0.06 & 0.05 & 0.19 & 0.17 & 0.13 & 0.17 & 0.23 & 0.20 & 0.12 \\
\hline W-KStar & 0.00 & -0.06 & 0.15 & 0.17 & 0.21 & 0.11 & 0.20 & 0.14 & 0.22 & 0.13 \\
\hline W-J48 & 0.00 & -0.06 & 0.22 & 0.25 & 0.25 & 0.30 & 0.26 & 0.25 & 0.24 & 0.19 \\
\hline Model-Average & 0.00 & -0.01 & 0.13 & 0.19 & 0.14 & 0.18 & 0.18 & 0.17 & 0.17 & \\
\hline
\end{tabular}


TABLE 7

ROC Area - Cynophobia Dataset

\begin{tabular}{|c|c|c|c|c|c|c|c|c|c|c|}
\hline $\begin{array}{l}\text { Selected } \\
\text { Attributes }\end{array}$ & ZeroR & OneR & JRip & $\mathrm{J} 48$ & REPTree & Naive Bayes & IBk & SMO & Bagging & $\begin{array}{l}\text { Attributes } \\
\text { - Average }\end{array}$ \\
\hline All & 0.50 & 0.51 & 0.53 & 0.57 & 0.55 & $\underline{0.73 \mathrm{v}}$ & 0.60 & 0.57 & $0.67 \mathrm{v}$ & 0.58 \\
\hline CFS & 0.50 & 0.59 & 0.58 & 0.55 & 0.53 & 0.69 & 0.68 & 0.64 & 0.62 & 0.60 \\
\hline InfoGain & 0.50 & 0.57 & 0.57 & 0.56 & 0.58 & 0.63 & 0.63 & 0.60 & $0.63 \mathrm{v}$ & 0.58 \\
\hline W-IBK & 0.50 & 0.47 & 0.52 & 0.60 & 0.56 & 0.60 & 0.62 & 0.61 & 0.56 & 0.56 \\
\hline W-KStar & 0.50 & 0.47 & 0.60 & 0.57 & 0.56 & 0.64 & 0.60 & 0.57 & 0.64 & 0.57 \\
\hline Model-Average & 0.50 & 0.50 & 0.57 & 0.58 & 0.54 & 0.64 & 0.62 & 0.58 & 0.60 & \\
\hline
\end{tabular}

TABLE 8

F- Measure - Cynophobia Dataset

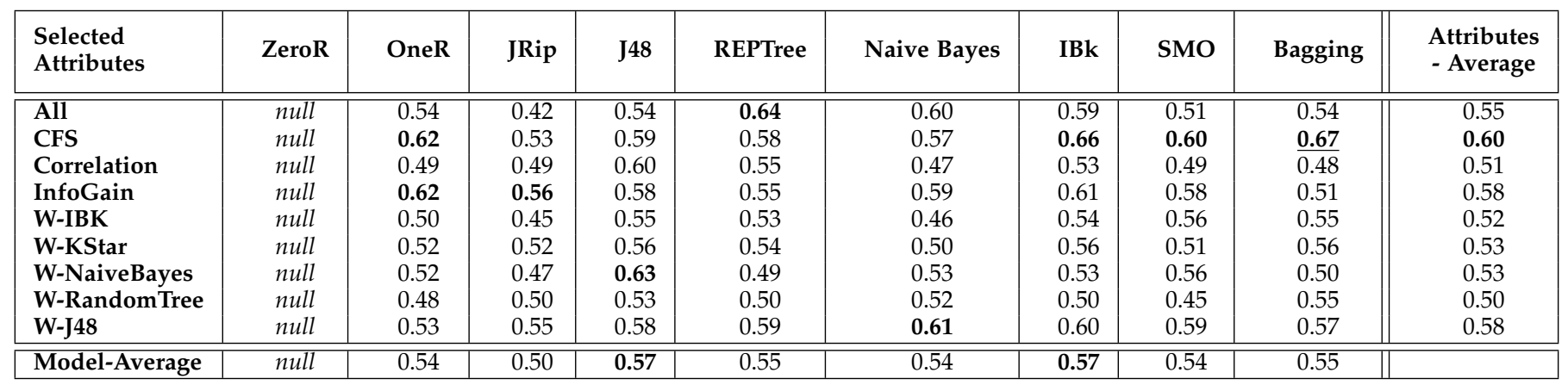

TABLE 9

Correctly Classified Instances Percentage - Astraphobia Dataset

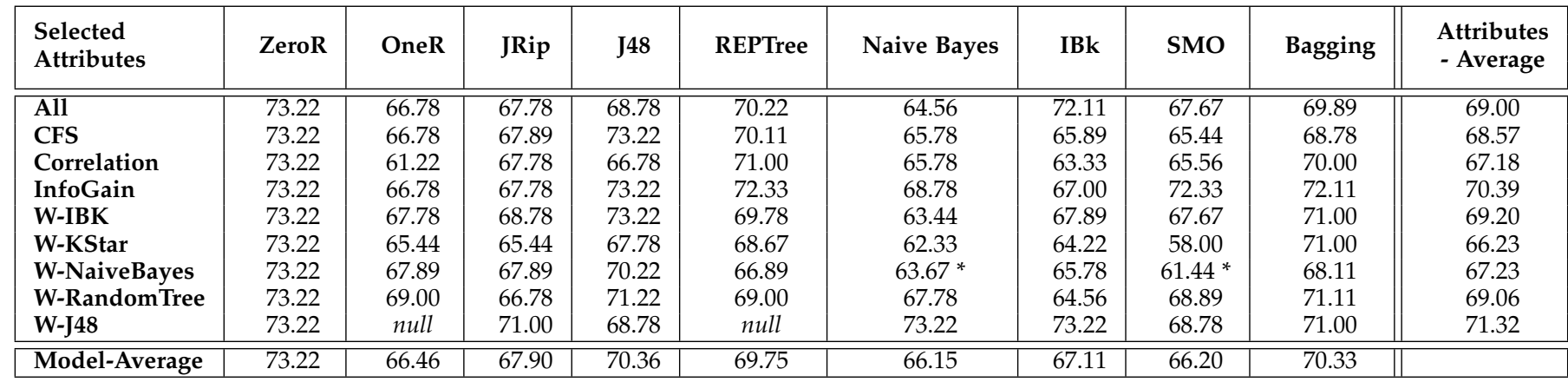

TABLE 10

Kappa Statistic - Astraphobia Dataset

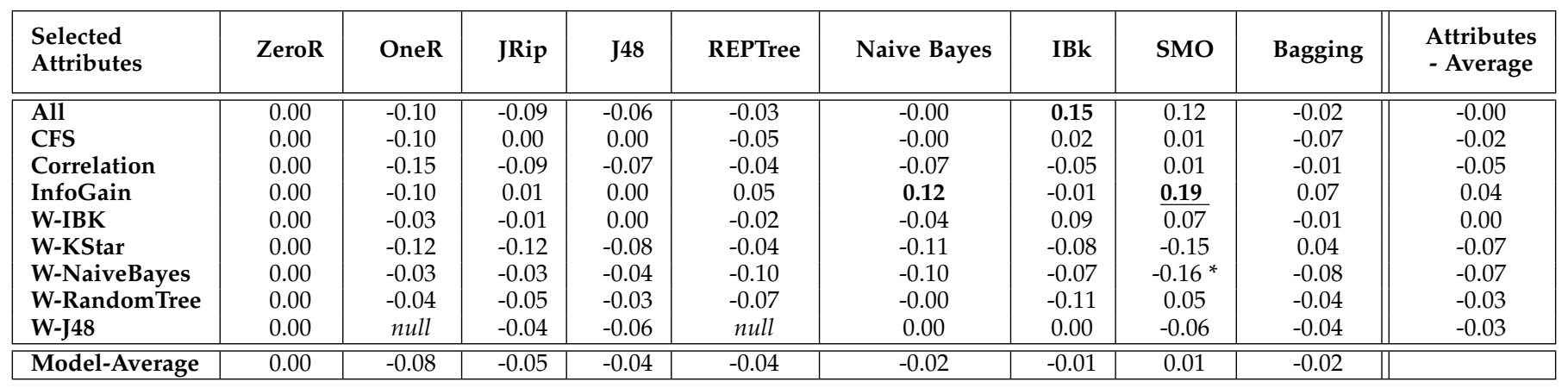


TABLE 11

ROC Area - Astraphobia Dataset

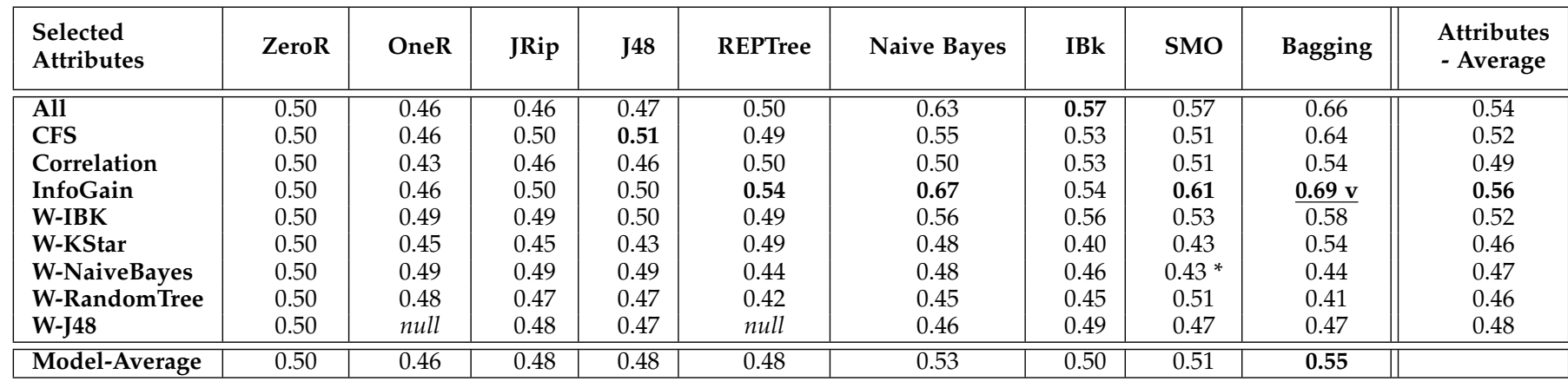

TABLE 12

F- Measure - Astraphobia Dataset

\begin{tabular}{|l|c|c|c|c|c|c|c|c|c||c|}
\hline $\begin{array}{l}\text { Selected } \\
\text { Attributes }\end{array}$ & ZeroR & OneR & JRip & J48 & REPTree & Naive Bayes & IBk & SMO & $\begin{array}{c}\text { Bagging } \\
\text { Attributes } \\
\text { - Average }\end{array}$ \\
\hline \hline All & 0.84 & 0.80 & 0.80 & 0.80 & 0.82 & 0.78 & 0.82 & 0.78 & 0.82 \\
CFS & 0.84 & 0.80 & 0.79 & $\underline{\mathbf{0 . 8 4}}$ & 0.82 & 0.78 & 0.78 & 0.77 & 0.81 \\
Correlation & 0.84 & 0.75 & 0.80 & 0.78 & 0.83 & $0.79 *$ & 0.76 & 0.77 & 0.82 \\
InfoGain & 0.84 & 0.80 & 0.80 & $\mathbf{0 . 8 4}$ & 0.83 & 0.80 & 0.79 & 0.82 & 0.83 \\
W-IBK & 0.84 & $0.80 *$ & 0.81 & $\mathbf{0 . 8 4}$ & 0.82 & 0.76 & 0.79 & 0.79 & 0.83 \\
W-KStar & 0.84 & 0.79 & 0.78 & 0.79 & 0.81 & 0.75 & 0.77 & 0.71 & 0.83 \\
W-NaiveBayes & 0.84 & 0.80 & 0.80 & 0.82 & 0.80 & $0.77 *$ & 0.79 & $0.75 *$ & 0.80 \\
W-RandomTree & 0.84 & 0.81 & 0.79 & 0.83 & 0.81 & 0.80 & 0.78 & 0.80 & 0.83 \\
W-J48 & 0.84 & null & 0.83 & 0.80 & null & 0.84 & 0.84 & 0.80 & 0.83 \\
\hline Model-Average & 0.84 & 0.79 & 0.80 & 0.82 & 0.82 & 0.78 & 0.79 & 0.78 & 0.80 \\
\end{tabular}

independent CFS evaluated attributes. The other two combinations are Naive Bayes model with All attributes and ensemble learning technique Bagging with scheme independent evaluator CFS calculated attributes. On average, CFS and W-J48 determined attributes performed well. The learning models: J48, Naïve Bayes, and IBk also worked adequately, on average. CFS attributes: Age, TimeNeutral, DistanceCyno, DistO2_BothCyno and DSMAstraphobia. W-J48 parameters: VRPresence and DSMAstraphobia.

Analyzing prediction classifiers for Astraphobia, study reveals that not a single combination of learning model and subset of attributes perform significantly for correctness, accuracy, agreeability, and diagnostic separability. However, the ranking based evaluator InfoGain make eminent combinations with different models for metrics "Correctly Classified Instances Percentage", Kappa Statitics, ROC Area and F-measure. InfoGain evaluates VelocityNeutral, TimeNeutral, VelocityBoth, TimeBoth, TimeCyno, DistanceNeutral, VelocityAstra, Age and DSMCynophobia.

\section{Conclusion}

The conclusions supported by this research analysis are as follows:

- The phobic stimulus in VRE usually elicited reactions of the participants with phobia in real life. Participants agreed they felt safe and responded much better in VR phobic stimulus environments comparatively confronted in real.
- The participants also reported that the audio-visual stimuli evoked better reactions than any single stimulus.

- The results discovered that Cynophobia (dog phobia) and Astraphobia (Thunder-lightning phobia) are highly correlated. As observed, participants with a thunder-lightning phobia are also identified with dogs phobia. The participants mentioned that these phobias are analogous due to audio-visual perceptions.

- The results verified the study that phobia problems affect the perceptions of 'Distance' and 'Time' [18], [17].

- The research outcomes project that Sense of Presence in VRE impacts for determining Cynophobia.

- The critical analysis indicates that the difference in oxygen levels in the presence of the phobic stimulus and multiple stimuli could be an effective physiological measure for phobia assessment.

\section{ACKNOWLEDGMENTS}

We cordially thank Computer Engineering Department, Research Lab, and Raja Muzammil (Teaching Fellow) for their support in conducting Research Experiments. 


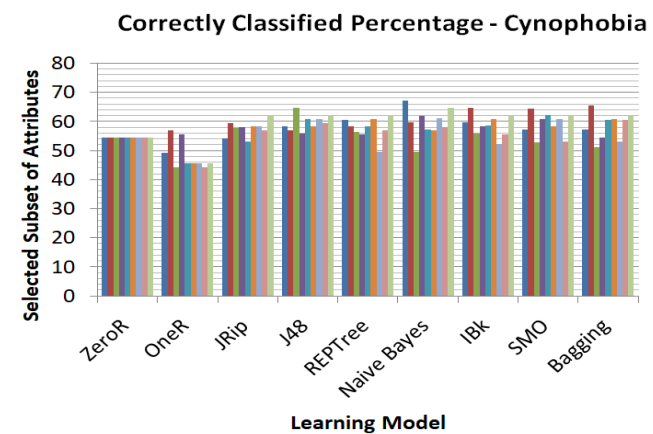

(a)

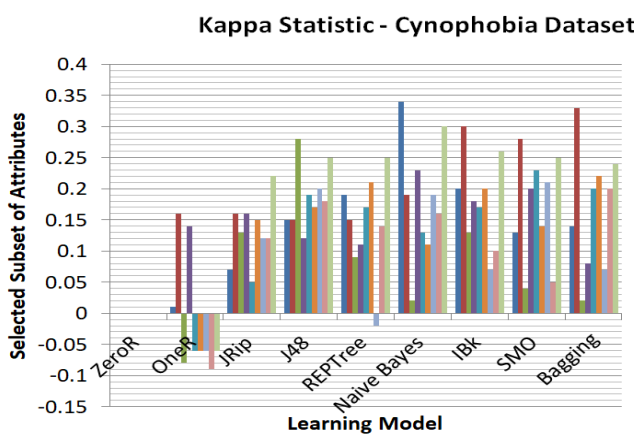

(b)

ROC Area - Cynophobia Dataset

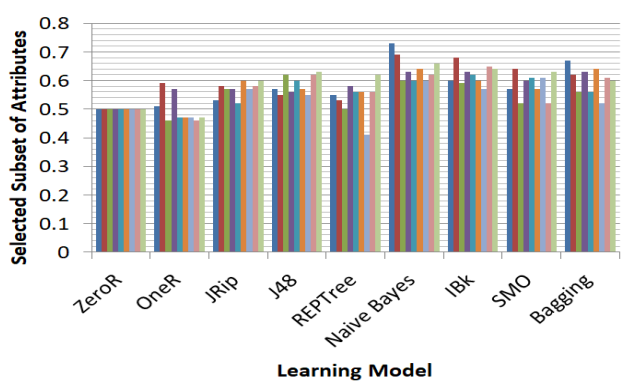

(c)

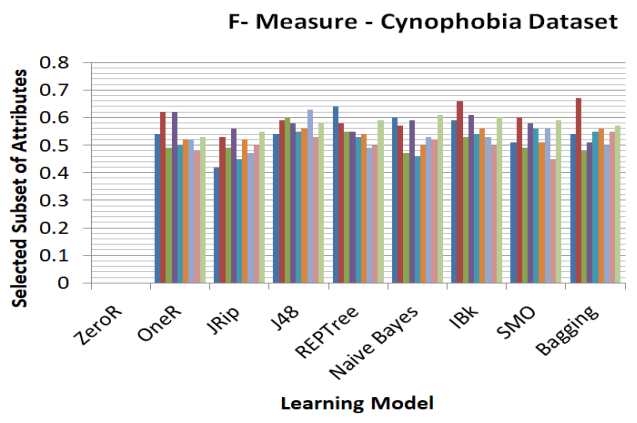

(d)

Fig. 10. Graphical Model Performance Comparison - Cynophobia

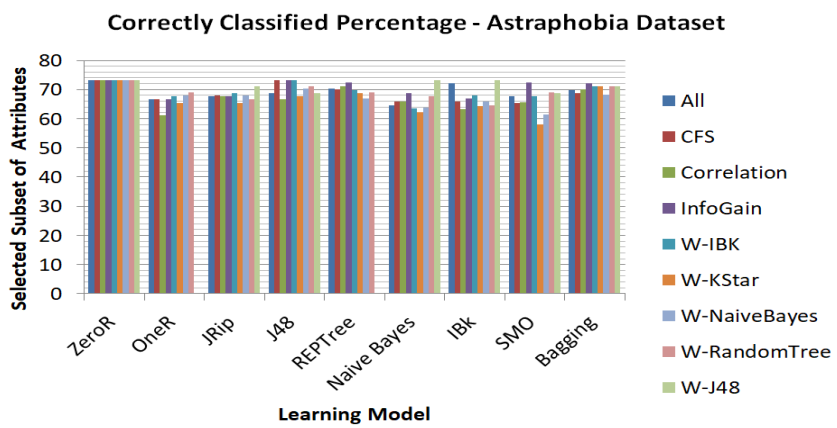

(a)

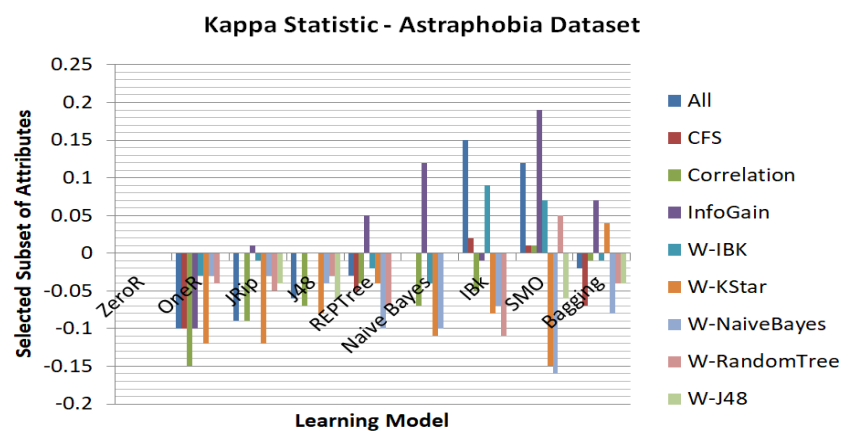

(b)

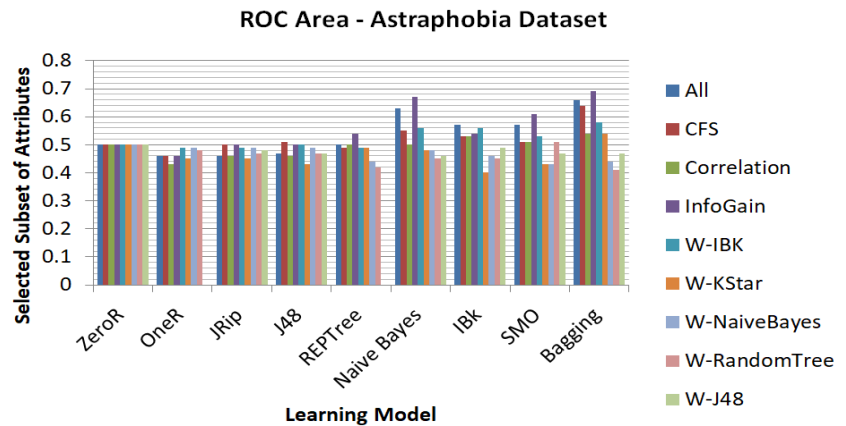

(c)

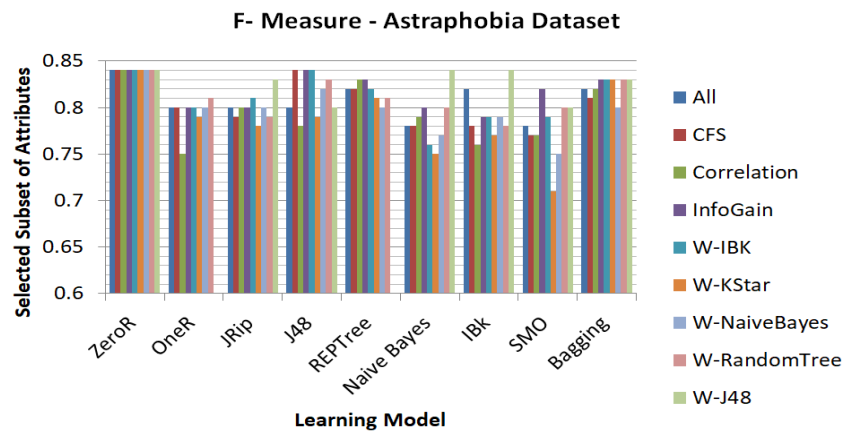

(d)

Fig. 11. Graphical Model Performance Comparison - Astraphobia 


\section{REFERENCES}

[1] M. Roser, Hannah Ritchie, "Mental health," https:// ourworldindata.org/mental-health, 2018, accessed: 2018-10-18

[2] A. P. Association, Diagnostic and Statistical Manual of Mental Disorders. American Psychiatric Association (APA), 2013.

[3] T. R. I. Hirschtritt Mathew E., "Digital technologies in psychiatry: Present and future," FOCUS, vol. 16, no. 3, pp. 251-258, 2018.

[4] T. R. Insel, "Digital phenotyping: a global tool for psychiatry," World Psychiatry, vol. 17, no. 3, pp. 276-277, 2018.

[5] M. J. van Bennekom, P. P. de Koning, and D. Denys, "Virtual reality objectifies the diagnosis of psychiatric disorders: A literature review," Frontiers in Psychiatry, vol. 8, p. 163, 2017.

[6] D. Freeman, S. Reeve, A. Robinson, A. Ehlers, D. Clark, B. Spanlang, and M. Slater, "Virtual reality in the assessment, understanding, and treatment of mental health disorders." Psychological Medicine, vol. 47, no. 14, pp. 2393-2400, 2017.

[7] M. A. Gigante, “Virtual Reality: Definitions, History and Applications," pp. 3-14, 1993.

[8] Statista, "Virtual reality (vr) - statistics \& facts," https://www.statista.com/topics/2532/virtual-reality-vr/, accessed: 2019-1-17.

[9] T.-A. Le and D. Beidel, "Psychometric properties of a social skills assessment using a virtual environment," Journal of Psychopathology and Behavioral Assessment, vol. 39, no. 2, pp. 230-240, 2017.

[10] T. F. Wechsler, F. Kümpers, and A. Mühlberger, "Inferiority or even superiority of virtual reality exposure therapy in phobias?-a systematic review and quantitative meta-analysis on randomized controlled trials specifically comparing the efficacy of virtual reality exposure to gold standard in vivo exposure in agoraphobia, specific phobia, and social phobia," Frontiers in Psychology, vol. 10, 2019.

[11] C. Botella, J. Fernández-Álvarez, V. Guillén, A. García-Palacios, and R. Baños, "Recent progress in virtual reality exposure therapy for phobias: A systematic review," Current Psychiatry Reports, vol. 19, 2017.

[12] M. C. Mishkind, A. M. Norr, A. C. Katz2, and G. M. Reger, "Review of virtual reality treatment in psychiatry: Evidence versus current diffusion and use," Curr Psychiatry Rep, vol. 19, no. 11, p. 80, 2017.

[13] C. Suied, G. Drettakis, O. Warusfel, and I. Viaud-Delmon, "Auditory-visual virtual reality as a diagnostic and therapeutic tool for cynophobia," CYBERPSYCHOLOGY, BEHAVIOR, AND SOCIAL NETWORKING, vol. 16, no. 2, pp. 145-152, 2013.

[14] E. Carl, A. T. Stein, A. Levihn-Coon, J. R. Pogue, B. Rothbaum, P. Emmelkamp, G. J. Asmundson, P. Carlbring, and M. B. Powers, "Virtual reality exposure therapy for anxiety and related disorders: A meta-analysis of randomized controlled trials," Journal of Anxiety Disorders, vol. 61, pp. 27-36, 2019.

[15] L. R. Valmaggia, L. Latif, M. J. Kempton, and M. Rus-Calafell, "Virtual reality in the psychological treatment for mental health problems: An systematic review of recent evidence," Psychiatry Research, vol. 236, pp. 189 - 195, 2016. [Online]. Available: http://www.sciencedirect.com/science/article/pii/S01651781163002

[16] I. Viaud-Delmon, F. Znaïdi, N. Bonneel, C. Suied, O. Warusfel, N. Khoa-Van, and G. Drettakis, "Auditory-visual virtual environments to treat dog phobia," in The Seventh International Conference on Disability, Virtual Reality and Associated Technologies with ArtAbilitation 2008. Porto, Portugal: ICDVRAT/University of Reading, UK;, 2008, pp. 119-124, session III - Virtual Reality Methodologies I. [Online]. Available: https://hal.inria.fr/inria00606817

[17] M. Taffou, R. Guerchouche, G. Drettakis, and I. Viaud-Delmon, "Auditory-visual aversive stimuli modulate the conscious experience of fear," Multisensory Research, vol. 26, no. 4, p. 347-370, 2013.

[18] M. Taffou and I. Viaud-Delmon, "Cynophobic fear adaptively extends peri-personal space," Frontiers in Psychiatry, vol. 5, p. 122, 2014

[19] N. Hnoohom and S. Nateeraitaiwa, "Virtual reality-based smartphone application for animal exposure," in 2017 International Conference on Digital Arts, Media and Technology (ICDAMT), March 2017, pp. 417-422.

[20] J. SW, G. M, v. W. HC, and K. B. MN, "Head-mounted virtual reality and mental health: Critical review of current research." JMIR Serious Games., vol. 6, no. 3, 2018.
[21] A. Y. Tychkov, D. S. Chernyshov, N. S. Bofanova, A. K. Alimuradov, D. L. Ovchinnikov, and A. M. Sotnikov, "Virtual reality implementation for assessment and treatment of phobic anxiety disorders," in 2021 5th Scientific School Dynamics of Complex Networks and their Applications (DCNA), 2021, pp. 202-205.

[22] K. Wood, A. J. Uribe Quevedo, L. Penuela, S. Perera, and B. Kapralos, "Virtual reality assessment and customization using physiological measures: A literature analysis," in Symposium on Virtual and Augmented Reality, ser. SVR'21. New York, NY, USA: Association for Computing Machinery, 2021, p. 64-73. [Online]. Available: https://doi.org/10.1145/3488162.3488228

[23] H. Buhl, "Instruction manual universal vr remote controller," http://v3.hamiltonbuhl.com/hammanual/Universal\%20VR\%20Remote $\% 20$ Co Manual-d10.pdf, accessed: 2019-10-15.

[24] Google, "Quickstart for google vr sdk for unity with android," https://developers.google.com/vr/develop/unity/get-startedandroid, accessed: 2019-10-15.

[25] S. E. Corporatio, "Epson exceed your vision," https://www5.epsondevice.com/en/information/technical_info/gyro/, accessed: 2020-09-14.

[26] Oracle, "Java se development kit 8 downloads," https://www.oracle.com/technetwork/java/javase/downloads/jdk8downloads-2133151.html, accessed: 2019-10-15.

[27] Google, "android studio," https://developer.android.com/studio, accessed: 2019-10-15.

[28] U. Technologies, "Unity for all," https://unity.com/, accessed: 2019-10-15.

[29] Microsoft, "Code editing. redefined." https://code.visualstudio.com, accessed: 2019-10-15.

[30] Blender, "blender," https://www.blender.org/, accessed: 2020-0408.

[31] A. Munir, "Research survey - diagnosis of phobic anxiety disorders using vr environment," https://docs.google.com/forms/d/e/1FAIpQLSeqiEPEqMLbr5tZ

[32] A. P. Association, "Online assessment measures," https://www.psychiatry.org/psychiatrists/practice/dsm/educationalresources/assessment-measures, accessed: 2020-01-12.

[33] WekaMOOC, "Data mining with weka (5.1: The data mining process )," https: / /www.youtube.com/watch?v=1Hg2JD2DYsI\&list=PLm4W7_iX_v4NqP OKNVO4c_cPD\&index=26, accessed: 2021-08-13.

Alina Munir has earned B.Sc and M.Sc degrees in Computer Engineering from the University of Engineering and Technology, Lahore. She won an Honorary Mention in IEEE ComSoc Student Competition - 2021. She 257 interested in cutting-edge technologies for their impact on life.

Dr. Yasir Saleem is an Associate Professor at the University of Engineering and Technology, Lahore. He has 15+ years of teaching experience at Graduate and Post-Graduate levels. He is interested in applying emerging Engineering technologies for interdisciplinary domains. 